\title{
A New Virtual Reality Environment Used for e-Learning
}

\author{
Zhenbo Li ${ }^{1,3}$, Jun Yue ${ }^{2}$, David Antonio Gómez Jáuregui ${ }^{1}$ \\ 1.Institut TELECOM ; TELECOM \& Management SudParis \\ 9 rue Charles Fourier, 91011 Evry Cedex, France \\ 2. Management College, LuDong University \\ Yantai, Shandong, 264025, China \\ 3. School of Computer Science and Technology, Shandong University \\ Jinan, Shandong, 250061, China \\ zhenboli@gmail.com; yuejuncn@sina.com; David.Gomez@IT-SudParis.eu
}

\begin{abstract}
Virtual reality has been successful used in real estate, urban planning and video games etc. In this paper, we proposed to build an interactive virtual eLearning environment using virtual reality technologies. With the help of Multi-Modal user interface, students can control their avatars to interactively communicate with virtual teachers and environment. This interactive e-Learning environment can increase the autonomy of students and enhance students' interest in learning.
\end{abstract}

\section{Introduction}

With more than ten years development, e-Learning has changed the traditional teaching-learning mode gradually. Nowadays, people are required to learn all their lives. E-Learning system builds digital environment using multimedia technology, Internet etc. This can help people learn what they wanted on their own initiative. Study can happen at anywhere and anytime. Traditional passive learning is replacing by engaged learning.

One of the tasks for an e-Learning system is how it can attract the audience. This is related with learning effect directly. In order to attract more audience, multimedia including text, image, video and audio is wildly used in e-Learning system this moment. This can increase learning passion at a certain extent. In this paper we proposed to build an immersed environment and use Multi-Modal user interface to attract audience in e-Learning.

Virtual Reality (VR) is an integrated technique involved computer graphics, human-computer interaction and artificial intelligence etc. Its object includes "3I" - Immersion, Interaction and Imagination (figure 1). Virtual reality tries to use computer to create $3 \mathrm{D}$ virtual world, people can experience in the virtual world as in real world.

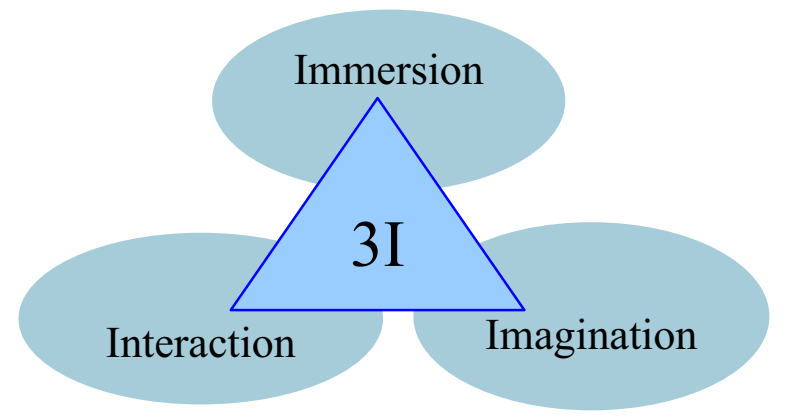

Figure 1. The object of virtual reality system

Virtual reality has been successful used in real estate, advertisement, urban planning and video games etc. With the development of computer hardware, it can be possible using a normal personal computer to implement a complex virtual environment [1, 2, 3]. To combine virtual reality with e-Learning system, a complete immersed virtual world can be constructed for e-Learning. People can live at the virtual community. Thus network space is changed to be a society space. Virtual community is used to increase interaction and immersion in e-Learning. This mode can help attract audience in e-Learning and improve learning effect.

The rest of this paper is organized as follow: in section 2, we introduced the related works about virtual reality in e-Learning. We will introduce to construct immersed virtual environment in section 3 . 
In section 4, we will present the work of using Multi-Modal user interface to help immersion and interaction feeling in virtual world. Conclusions are given in section 5 .

\section{Related Works}

Some researchers have studied the relationship between virtual reality and e-Learning. ShangZhen Yang et al.[4] discussed the application and influence of virtual reality in e-Learning. Min Chen et al.[5] discussed the method of building distributed virtual environment and implement e-Learning in the virtual environment. She also gave a Client-Server distributed virtual environment including e-Learning system. Jiejie Zhe et al.[6] described a virtual multimedia classroom system(VMCS). They pictured the use and function of the system. Also they analyzed the technologies used for constructing virtual classroom like LOD (levels of details) in his work. Zhigeng Pan et al.[7] explores educational uses of Virtual Learning Environment (VLE) concerned with issues of learning, training and entertainment. They analyze the state-ofart research of VLE based on virtual reality and augmented reality. They described some examples for the purpose of education and simulation. They proved that VLE can be means of enhancing, motivating and stimulating learners' understanding of certain events, especially those for which the traditional notion of instructional learning have proven inappropriate or difficult.

Bouras et al. [8][9] have done a lot of research on embedding education in distributed virtual environments. They tried to build distributed virtual environments to allow students and staff to meet in social shared spaces and engage in on-line real-time seminars and tutorials. They discussed some key technologies in their work. Also they presented system architecture to support use of collaborative virtual environments in e-Learning. Teresa et al.[10] presented their work of development a Collaborative Learning Environment with Virtual Reality(CLEV-R). Their web-based system uses virtual reality and multimedia and provides communication tools to support collaboration among students. Sester et al. [11] gave an overview on the concepts of e-Learning environment and described the technical structure of web-based geodata visualization and interaction. Ouyang XUN et al. [12] provided an overview of using current virtual reality technology to construct virtual environments for real time based e-Learning. They presented a virtual reality-based e-Learning framework example that aims to facilitate the construction of realistic and interactive virtual learning environments. Their system used innovative techniques for constructing virtual learning environments for selected teaching purpose.

\section{Immersed Virtual e-Learning Environment}

\subsection{Virtual Community}

Content is the most important part of e-Learning, while the e-Learning form can help the learning effect. Nowadays, virtual community like Facebook (www.facebook.com) is spread in the world with the speedy development of Internet. More and more people are attracting to make friends, get knowledge in the virtual community. At the same time, 3D virtual community likes SecondLife (www.secondlife.com), MyLife3D (www.mylife3d.com) attract many people to experience their virtual life.



Figure 2. Virtual class in SecondLife (https://blogs.secondlife.com)

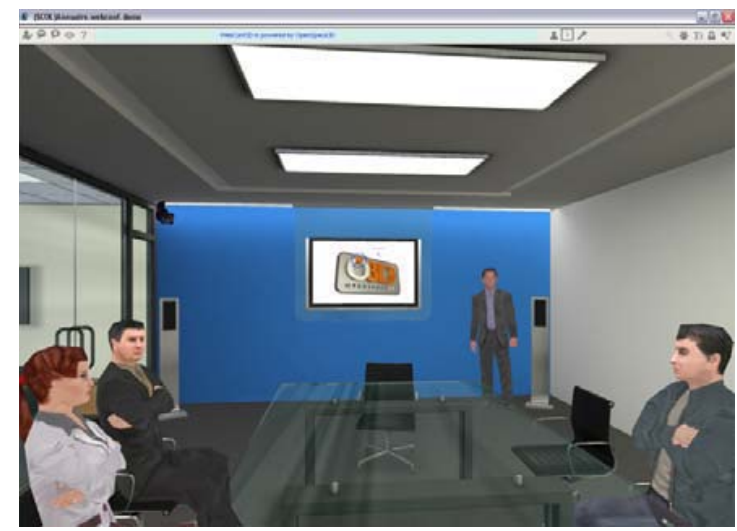

Figure 3. Virtual conference in MyLife3D (www.webconf3d.com) 
In $3 \mathrm{D}$ virtual community, each people have an avatar. They can communicate with other peoples as well as do activities as they do in their daily life. E-Learning can also be done in the virtual community. People can attend virtual class, virtual conference etc. They can discuss with virtual teachers and other peoples. People can choose their study resources, study form and study plan. This more immersion and interactive virtual community is becoming one of the future development direction of e-Learning.

\subsection{Immersed Virtual Museum e-Learning Environment}

In immersed virtual e-Learning environment, people can control their avatars to communicate with other peoples and learn the courses they want to learn. They can play video games, watch movies and do virtual excises in virtual environment. This can easy attract people's interest in learning. The immersed virtual eLearning environment needs the support of hardware. Different hardware level can reach different reality effect. In our work, we are using Helmet-Mounted Displayer (HMD) to implement an immersed virtual museum e-Learning environment.

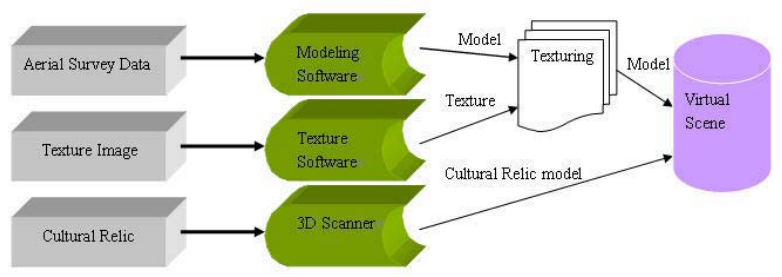

Figure 4. The framework of constructing virtual environment

Immersed virtual environment is a complex system. Constructing a virtual e-Learning environment includes two parts, building virtual scene and define events. Because virtual environment is highly dependent on models, it is important to deal with 3D models. Too much model details will reduce the simulation effect, especially when the models are rendering through Internet (e.g. using VRML). We always need to balance the rendering effect and model details. Textures are normally used to replace model details in the system. One technique is to divide the models into small parts. Rendering can be done simultaneity in the pipeline.

Cultural relic models are important for our virtual museum e-Learning system. People always want to observe and learn the details of cultural relic. In order to guarantee the quality of cultural relic models, we use 3D scanner to model the cultural relic. High quality $3 \mathrm{D}$ cultural relic models are got through scanning.

Because 3D models cost lots of rendering time and transferring time through Internet, LOD (levels of details) is used in our environment to rendering 3D cultural relic models according the distance and viewpoint direction of avatars. Coarse models are used when the avatars are far from the cultural relic models. Otherwise precision models are used. Visibility estimation is another way to improve rendering effect of virtual environment. Models will not render if out of user's visual field. Also textures and lights are important for the reality of virtual e-Learning environment.

As we use HMD as our displayer to improve immersion feeling (figure 5), two parallax images will be computed and transmit to the two glasses of HMD using formula 1. Stereo image will display after people syncretized the parallax images together using their brain.

$$
e=2 \operatorname{dtg}(\beta / 2)(i+1) / i
$$

Here, $e$ is the distance of left-right viewpoint, $d$ is the distance of viewpoint to projection screen, $\beta$ is visual angle(HVA). $i$ is depth information. $e$ and HVA are the two parameters need to tune for stereo results.

\section{Multi-Modal User Interface}

User interface is important for the experience of virtual e-Learning environment. For the current interactive system, graphical user interface and twodimensional interaction devices such as keyboard and mouse are wildly used. They can greatly facilitate nonprofessional users. People no longer need to remember a large number of commands. Human computer interface has gradually shifted from the traditional people to adapt to the complex computers to computer adapt to users.

For the immersed virtual e-Learning environment, the learning process is progressing in a $3 \mathrm{D}$ virtual world. Multimedia class contents are displayed in the virtual world. People need to interact with the 3D world. The 2D user interface and devices are not natural and difficult for the user immersing in 3D world. Thus 3D user interface need to development for the interaction. Normally this is a Multi-Modal user interface.

In our system, we use captured gestures as main interface to interact with the virtual e-Learning environment. Here, we try two ways to capture user's 
gestures, Data Glove+3D tracker and vision based motion capture from webcam. User's gestures are used to interact with virtual e-Learning environment. People can choose what they want to learn and communicate with avatars (teachers or other students) using gestures, voice and text. For example, people can grasp cultural relic models in virtual environment and observe cultural relic models normally this is forbidden in real life. Also he can hear and watch the introduction of the cultural relic. Tracker on the HMD can detect user's place and viewpoint direction in the virtual world. Voice feed back can be got through the earphone on the HMD.

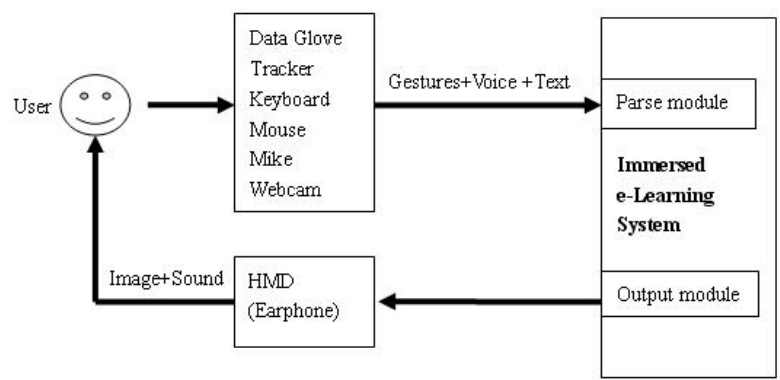

\section{Figure 5. Framework of Multi-Modal user} interface

Traditional e-Learning material (like cultural relic introduction etc.) can embed in the virtual e-Learning environment. The immersed virtual environment and Multi-Modal user interface can attract users' interest in e-Learning. Also the virtual environment can be constructed as Client/Server mode on the Internet.

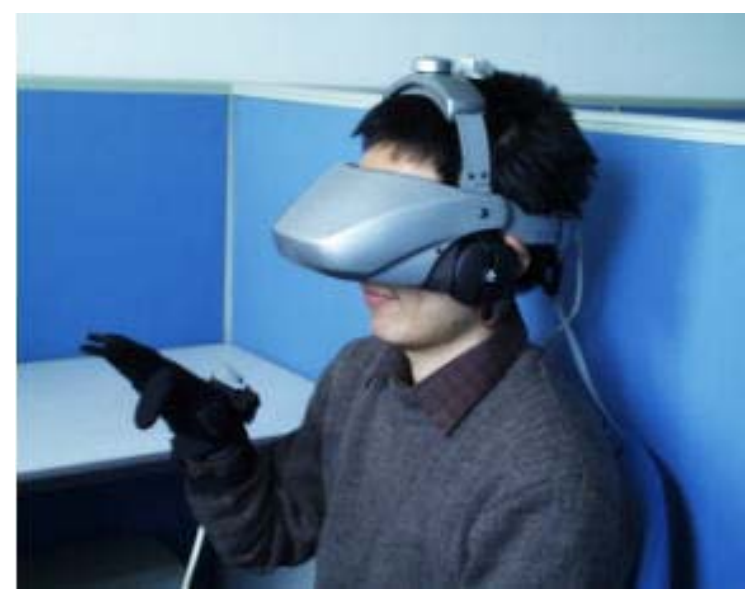

Figure 6. HMD and Data Glove are used for Multi-Model interface in the system.

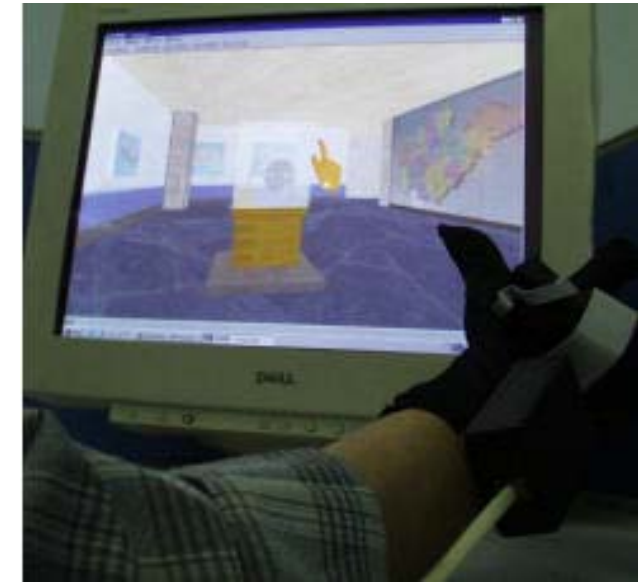

Figure 7. Exploring and learning in virtual museum

\section{Conclusion}

E-Learning is one of the emerging needs of the information age. Access to education is going to become crucial for the success of our information society, e-Learning is a good solution for modern teaching and learning. Except improving e-Learning materials, e-Learning form is also an important factor related with learning effect.

We proposed to build an immersed virtual eLearning environment and use Multi-Modal user interface in e-Learning in the paper. The system has good immersion and interaction ability. The immersed e-Learning system can make students more activity in learning process. Compare to traditional e-Learning system, immersed virtual e-Learning environment can maintain students' interest and keep them engaged and motivated in their learning.

One of the future works is to research how to combine Multi-Modal user interface and more eLearning materials into virtual community.

\section{References}

[1] Zhenbo LI, Xiangxu MENG, Hui XIANG. The Research And Implementation of Constructing Complicated Interactive Virtual Scenes. Journal of System Simulation. (2002). Vol.14,No.9. pp:1183-1187.

[2] Kun ZHOU, YiYing TONG, ZhiGeng PAN, JiaoYing SHI. VECW: A Virtual Environment Construction and Walkthrough System. Journal of Computer-Aided Design \& Computer Graphics. 2000 Vol.12, No.5. pp:355-359.

[3] Xin ZHENG, Enhua WU. Model Transformation and Simplification from VRML to MultiGen/Vega Practice in a 
Virtual System of University of Macao. Journal of System Simulation.(2001) Vol.13. pp:440-442.

[4] Shangzhen YANG, Dan LIU. E-learning and Visual Reality. China Modern Education Equipment. (2007) Vol.7. pp:16-17,42.

[5] Min CHEN, Huidi LUO, Fang QU. The Design and Implementation of e-Learning in Distributed Virtual Environment. Journal of Educational Technology \& Communication. (2002).Vol.1.

[6] Jiejie ZHU, Weihua HU, Zhigeng PAN. Design and Implementation of Virtual Multimedia Classroom. Journal of Computer-Aided Design \& Computer Graphics. (2004) Vol.1. pp: 74-79.

[7] Zhigeng PAN., et al.: Virtual reality and mixed reality for virtual learning environments. Computers \& Graphics. (2006). Vol. 30, No.1, pp:20-28.

[8] Bouras C., Philopoulos A., Tsiatsos T H. E-learning Through Distributed Virtual Environments[J]. Journal of Network and Computer Applications, 2001, 24(3): 175-199.

[9] Bouras C., Hornig G., Triantafillou V., Tsiatsos T.. Architectures Supporting e-Learning Through Collaborative Virtual Environments:The case of INVITE ICALT (2001),pp:13-16.

[10] Monahan T., McArdle G., Bertolotto M.. Virtual Reality for Collaborative E-Learning. Elsevier Journal of Computers and Education, (2008). pp: 1339-1353.

[11] Sester. M., Katterfeld. C.. Desktop Virtual Reality in Elearning Environments, ISPRS Congress, Istanbul, Commission 6, (2004).

[12] Ouyang XUN, Kharaz AHMAD, Thorn RICHARD. Constructing Virtual Environments for Real Time E-learning International Journal of Computer Science and Network Security, (2006).VOL.6 No.5A. pp. 1-5. 